\title{
Combined culture experiment of mouse bone marrow mesenchymal stem cells and bioceramic scaffolds
}

\author{
XIN HUANG ${ }^{*}$, ZHENHAO CHEN*, GUANGLEI ZHAO, JINGSHENG SHI, GANGYONG HUANG, \\ FEIYAN CHEN, YIBING WEI, JUN XIA, JIE CHEN and SIQUN WANG
}

Department of Orthopedics, Huashan Hospital, Fudan University, Shanghai 200040, P.R. China

Received July 12, 2019; Accepted April 24, 2020

DOI: $10.3892 / e t m .2020 .9147$

\begin{abstract}
Articular cartilage injuries are common orthopedic conditions that severely affect the quality of life of patients. Tissue engineering can facilitate cartilage repair and the key points involve scaffolding and seed cell selection. Pre-experiments found a range of microstructures of bioceramic scaffolds suitable for chondrocyte adhesion and proliferation, and maintaining chondrocyte phenotype. Three-dimensional cultures of bone marrow mesenchymal stem cell (BMSC) scaffolds were implanted into mice. According to the shape of the bioceramic scaffolds and the implantation time in vivo, RNA sequencing was performed on the removed scaffolds to explore the molecular mechanism. The in vitro bone plate culture can induce differentiation of chondrocytes, making culture different to that produced in vitro. Implantation of scaffolds in vivo increases the expression of bone-related genes. The ceramic rod-like material was found to be superior to the disc shape, and the bone repair effect was more marked with longer implantation times. Gene Ontology analysis revealed that 'cell chemotaxis', 'negative regulation of ossification' and 'bone development' pathways were involved in recovery. It was further confirmed that BMSCs were suitable as seed cells for cartilage tissue engineering, and that the $\beta$-tricalcium phosphate scaffold maybe ideal as cartilage tissue engineering scaffold material. The present research provided new insights into the molecular mechanism of cartilage repair by BMSCs and bioceramic scaffolds. Bioinformatics analysis revealed that AMMECR1L-like protein, tumor necrosis factor-induced protein 2, inhibitor of nuclear factor-B kinase subunit and protein kinase $\mathrm{C}$ type and 'negative regulation of ossification'
\end{abstract}

Correspondence to: Professor Siqun Wang or Dr Jie Chen, Department of Orthopedics, Huashan Hospital, Fudan University, 12 Urumqi Middle Road, Jing'an, Shanghai 200040, P.R. China

E-mail: wsqun66@sina.com

E-mail: cj0120@126.com

*Contributed equally

Key words: bone regeneration, tissue engineering, tissue scaffold, mesenchymal stem cells, $\beta$-tricalcium phosphate and 'bone development' pathways may be involved in osteoblast maturation and bone regeneration.

\section{Introduction}

Articular cartilage injury is a common and frequent disease-related orthopedic condition (1). Inadequate and improper treatment can lead to osteoarthritis, pain and dysfunctional walking, which affect the quality of life of patients (2). Due to poor self-repairing ability of the articular cartilage, the current clinical treatment of articular cartilage defects is not satisfactory; in particular, repair of the damage to the full-thickness articular cartilage is a challenge for clinicians (3). Tissue engineering can facilitate cartilage repair, with numerous successful reports based on animal experiments $(4,5)$. Scaffold and seed cell selection are important factors affecting cartilage repair.

Bone marrow mesenchymal stem cells (BMSCs) are suitable cellular materials for articular cartilage repair. Cartilage differentiation is an intrinsic property of mesenchymal stem cells (6). Most in vitro and in vivo studies suggest that BMSCs have the potential to increase osteoinduction and osteogenesis (7-11). A study has demonstrated that seeding BMSCs on biocompatible scaffolds may be an effective method for treating nonunion fractures (12). Mesenchymal stem cells have the potential to home to damaged areas, which may enhance repair in two respects: i) Differentiation of tissue cells, specifically, the recovery of lost morphology and function; and ii) secretion of various biologically active factors, which have antiapoptotic effects and immunoregulatory functions, thereby creating an environment that stimulates the proliferation of endothelial progenitor cells and leads to subsequent repair (13). A clinical study has demonstrated the effectiveness of direct local BMSC delivery by injection in promoting bone regeneration (14). However, in large bone defects where a significant amount of bony tissue has been lost, the direct injection method was found to be ineffective for BMSC delivery and the commensurate acceleration of the bone healing process (15). Cartilage repair without a scaffold or soft support does not provide sufficient mechanical support, leading to insufficient cell enrichment in the repair area, hindering early weight bearing and an environment with insufficient pressure and nutritional support to induce cartilage repair (16). The bioceramic hard scaffold is prepared using a controlled microporous structure of $\beta$-tricalcium 
phosphate ( $\beta$-TCP) as the raw material (17). $\beta$-TCP is capable of triggering proliferation, migration and differentiation of the bone cells required for bone regeneration $(18,19)$. Porous ceramics have good biocompatibility and high mechanical strength, and their porous structure and degradation rate can be controlled according to the growth of the tissue (20). Physical microstructures, such as the bore and internal junction, can be regulated to facilitate cell adsorption and proliferation (15). High-porosity scaffolds facilitate the migration and proliferation of bone marrow cells in the scaffold, which is essential for the repair of osteochondral injury (21).

Previous experiments were conducted to determine a preliminary range of bioceramic scaffold microstructures suitable for promoting chondrocyte adhesion and proliferation and for maintaining the chondrocyte phenotype. The present study implanted a three-dimensional culture of BMSC scaffolds into mice and used RNA sequencing to explore the molecular mechanism of the bioceramic scaffolds on the basis of their shape and duration of implantation.

\section{Materials and methods}

Cell culture and flow cytometry. The 3rd generation BMSCs (CinoAsia Co., Ltd.) were digested using trypsin, after which $1 \times 10^{5} / \mathrm{ml}$ cell suspensions in DMEM/F12 (Gibco; Thermo Fisher Scientific, Inc.) were generated in an eppendorf tube. A total of $10 \mu$ l fluorescently-labelled CD34 (cat. no. ab8158), CD45 (cat. no. ab33923) and CD44 (cat. no. ab119348) antibodies (all, Abcam; all, 1:100) were added to $100 \mu \mathrm{l}$ of the cell suspension and incubated for $1 \mathrm{~h}$ at $37^{\circ} \mathrm{C}$. Flow cytometry was performed to detect individual cell markers (FACSMelody; Becton, Dickinson and Company; software, Flowjo, version 7.6; Becton, Dickinson and Company). BMSCs were counted and resuspended in complete DMEM/F12 supplemented with $10 \%$ fetal bovine serum and $1 \%$ senicillin-streptomycin solution (all, Gibco; Thermo Fisher Scientific, Inc.). Samples were cultured at $37^{\circ} \mathrm{C}$ and cell density was adjusted to $1 \times 10^{6}$ cells $/ \mathrm{ml}$.

Combined culture of BMSCs and bioceramic scaffolds. The $\beta$-TCP bioceramic material (purchased from Shanghai Bio-lu Biomaterials Co., Ltd.) was prepared and placed in a 24-well plate. First, $200 \mu 1$ of BMSCs suspended in DMEM/F12 (Gibco; Thermo Fisher Scientific, Inc.) were added to one side of the ceramic plate and incubated for $1 \mathrm{~h}$ at $37^{\circ} \mathrm{C}$. Subsequently, $200 \mu \mathrm{l}$ of the cell suspension was added to the reverse side, where it was incubated for another $1 \mathrm{~h}$ at $37^{\circ} \mathrm{C}$. DMEM/F12 was added to a total volume of $1 \mathrm{ml} /$ well and scaffolded cells were placed into medium for routine culture.

Bioceramic scaffolds implanted in mice. After 3 days of culture, the bioceramic scaffolds were planted into the femoral trochlea of the mice, where they remained for 3 or 6 days. The scaffolds were removed from the mice and crushed with small tweezers. A total of 40, six-week-old male mice (weight, 22-26g, Shanghai SLAC Laboratory Animal Co., Ltd.) were randomly divided into 5 groups (each, $n=8$ ). Animals were maintained at $25^{\circ} \mathrm{C}$, with a relative humidity of $40-70 \%$, under specific-pathogen free conditions. Animals were housed under a $12 \mathrm{~h}$ light/dark cycle with free access to food and water. Mice anaesthetized by intraperitoneal injection of $1 \%$ sodium pentobarbital $(50 \mathrm{mg} / \mathrm{kg})$. Following implantation of the ceramic material, the health and behavior of the mice were observed every day. Following removal of the ceramic material after 3 or 6 days, mice were intraperitoneally injected with an overdose $(1 \% ; 150 \mathrm{mg} / \mathrm{kg})$ of sodium pentobarbital for euthanasia. Mice with no breathing for $3 \mathrm{~min}$ and no corneal reflex were considered to be successfully euthanized.

Sequencing. Several small particles were placed in cell lysates $(0.2 \%$ triton $\mathrm{X}-100+5 \%$ RNase inhibitor), and stored at $-80^{\circ} \mathrm{C}$ for in vitro sequencing. The cultured ceramic material was digested with trypsin, and the library was sequenced. The sequences were grouped into controls (untreated mice; CONT); ceramic disc in vitro (MAT1); ceramic rod in vitro (MAT2); ceramic rod, 3 days in vivo (DAY3) and ceramic rod, 6 days in vivo (DAY6). Experiments in each group were performed in triplicate.

Ethical approval. All experiments were performed according to the principles outlined in the Guide for the Care and Use of Laboratory Animals and approved by the Ethics Committee of Huashan Hospital Affiliated to Fudan University (approval no. 2018 Huashan Hospital JS-098).

RNA database and sequencing. The cells cultured as described above were subjected to RNA sequencing, and each group was replicated three times. Cellular RNA was extracted and reverse transcribed into cDNA for PCR amplification. The PCR amplification reaction product was purified and subjected to quality control in a 96-well plate. The samples described above were measured by molar conversion, and each $2 \mathrm{nM}$ sample was diluted to $1: 1$. After dilution, $10 \mu \mathrm{l}$ of the sample was removed from the main tube and sent to the sequencing company for quality control with a 2100 QC bioanalyzer (Agilent Technologies, Inc.) before sequencing. The Hiseq2500 sequencer(Illumina, USA) was used, and the sequencing mode was set to a 2x150 bp read length with a $250 \mathrm{M}$ read number.

Raw data processing. The raw data were first processed using Trimmomatic (http://www.usadellab.org/cms/) to remove sequencing linker sequences and low quality read lengths. The remaining high-quality reads were aligned with the 25,014 genes of the Mus musculus genome using HiSat2 (http://daehwankimlab.github.io/hisat2/main/). The expression levels of each gene were quantified using featureCounts software, which produced raw count values for each sample. For raw data analysis, the counts of the sample genes were defined as $>1$, which was used as the base count value for the expressed genes. The read count was then normalized to the transcripts per kilobase million value for the $\log 2$ conversion with the 'newSCESet' function of the 'scater' package in R (https://www.r-project. org/). Principal component (PC) analysis, one-way ANOVA and hierarchical cluster analysis (HCA) using the 'prcomp', 'cor', 't.test' and 'cluster' functions were performed using the $\mathrm{R}$ 'cluster stats' package. Following analysis, a heatmap was generated with the R 'ComplexHeatmap' package and the PC analysis results were visualized using 'ggplot'.

Analysis of differentially expressed genes (DEGs). DEGs were identified by calculating the fold-change and P-values 

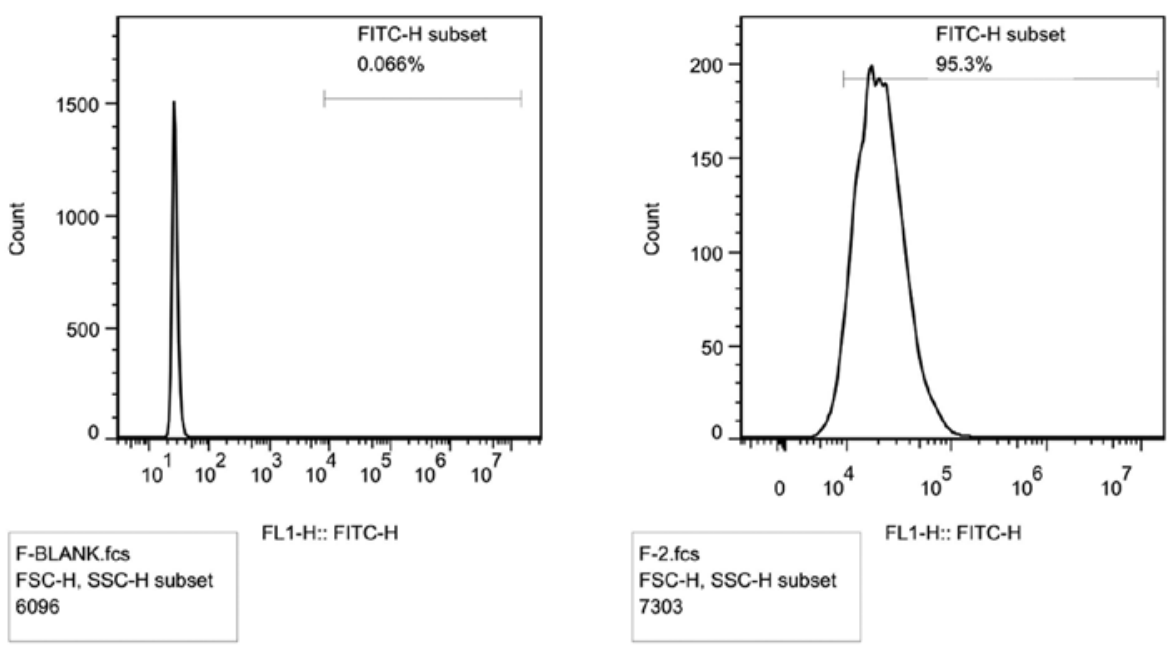

CD44
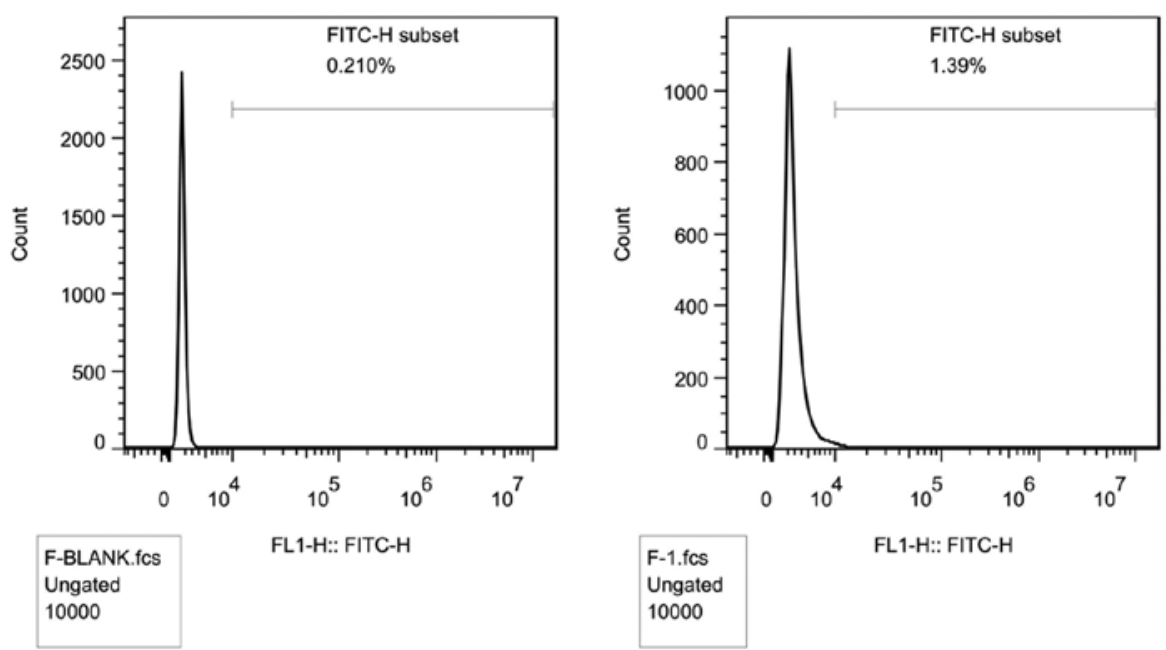

\section{CD34}
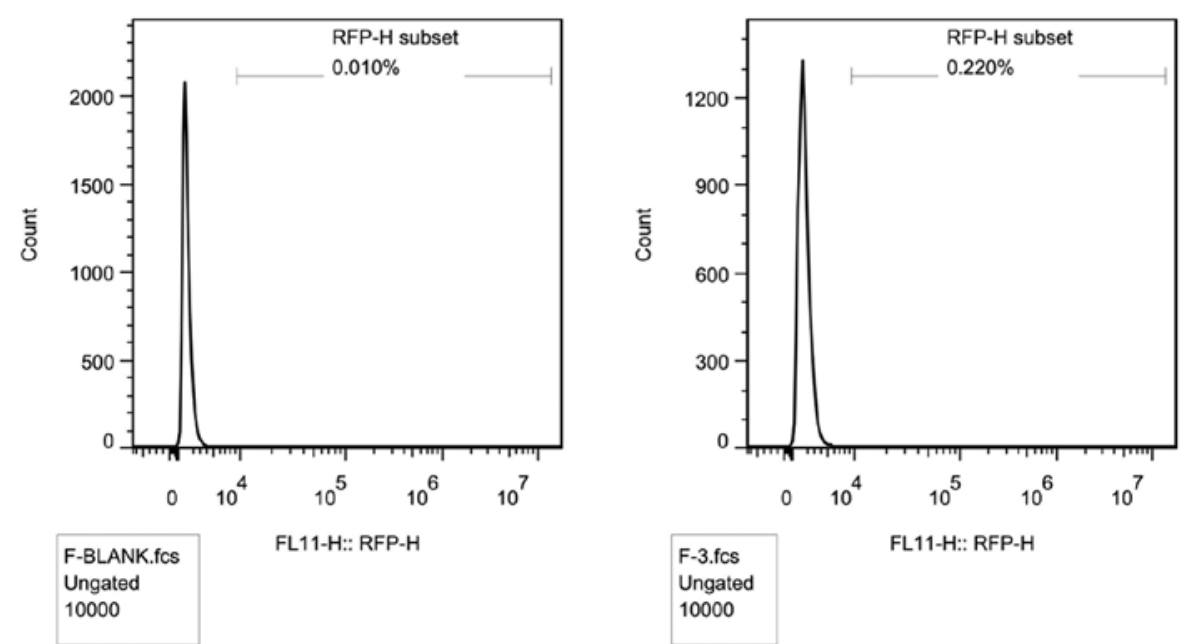

\section{CD45}

Figure 1. Expression of surface markers CD44 (95.3\%), CD34 (1.39\%) and CD45 (0.22\%) in bone marrow mesenchymal stem cells as determined by flow cytometry.

of the experimental and control groups. The multiples were modified to be $>$ two-fold; $\mathrm{P}<0.05$ was used as the standard for DEG selection and the 'stat' package in R was used. DEG intersections were determined with a 'Venn diagram' online tool (http://bioinformatics.psb.ugent.be/webtools/Venn/). Pathway analysis was performed using and Gene Ontology
(GO; http:/geneontology.org/) analysis was performed using the Database for Annotation, Visualization and Integrated Discovery (version 6.7; https://david.ncifcrf.gov/).

$m R N A$ sequencing analysis. The quality of the sequencing data was evaluated prior to data analysis using FastQC. 


\section{Results}

Cell identification. The cell surface antigens were detected by flow cytometry. The BMSC marker CD44 (95.3\%) was expressed, but not CD34 (1.39\%) or CD45 (0.22\%), indicating that the cells were BMSCs (Fig. 1).

RNA sequencing analysis. PC1 level analysis showed a good sample repeatability within the group, and the ceramic scaffold culture was superior compared with the CONT group. The degree of differentiation in three groups was measured by diffusion pseudotime analysis. Compared with MAT1 group, MAT2 group was farther from the CONT group in terms of the evolutionary trajectory, MAT2 was exhibited with a more differentiated state. Compared with the CONT group, the MAT2 group was superior to the MAT1 group (Fig. 2A). HCA revealed the characteristic genes of each group (Fig. 2B). Characteristic genes of the CONT group included transcription factor HES-1, ferritin light chain 2 and serine/threonine-protein phosphatase PP1-a catalytic subunit. Characteristic genes of the MAT1 group included neutrophil gelatinase-associated lipocalin (Lcn2), decorin (Dcn) and thrombospondin 2 (Thbs2). Characteristic genes of the MAT2 group included Lcn2, Dcn, Thbs2 and C-C motif chemokine ligand 7 (Ccl7).

Comparison between the top 10 upregulated and downregulated genes in the MAT1 and MAT2 groups compared with the CONT group found that the shared genes were serum amyloid A-3 protein (Saa3), Lcn2, nitric oxide synthase (Nos2) and nuclear receptor subfamily 4 group A member 1 (Tables I and II).

The DEGs between the MAT1 and MAT2 groups were compared, and the top 10 upregulated and downregulated genes are listed in Table III. Functional and pathway analysis of the DEGs was further performed (Fig. 3A). Three bone-related genes, NADH: Ubiquinone oxidoreductase subunit B6 (Ndufb6), ribosomal protein L17, pseudogene 9 (Rp117-ps9) and $\mathrm{Ccl}$, were selected for comparison (Fig. 3B).

Based on the DEG analysis, there were 1,274 DEGs in the MAT2 and CONT groups, 2,659 DEGs in the DAY3 and CONT groups and 1,785 DEGs in the DAY6 and DAY3 groups. With additional searches for genes with consistently changed expression compared with the CONT, MAT2 and the DAY3 and DAY6 groups, 10 genes were found to have increased expression levels: AMMECR1L-like protein (Ammecrll), tumor necrosis factor a-induced protein 2 (Tnfaip2), La Ribonucleoprotein 4B, Zinc Finger ZZ-Type And EF-Hand Domain Containing 1, Acid Phosphatase 2, inhibitor of nuclear factor $\kappa$-B kinase subunit $\varepsilon$ (Ikbke), protein kinase $\mathrm{C} \delta$ type (Prkcd), ATP Binding Cassette Subfamily A Member 1, CD44 Molecule and HIVEP Zinc Finger 2. A total of nine genes were found to have decreased expression levels:, including ferredoxin-1 (Fdx1), Eukaryotic Translation Initiation Factor 4E Binding Protein 1 (Eif4ebp1), insulin-like growth factor-binding protein 6 (Igfbp6), Solute Carrier Family 25 Member 4 (Slc25a4), Ribosomal Protein L14 (Rpl14), Actin Alpha 2, Smooth Muscle (Acta2), barrier-to-autointegration factor 1 (Banf1), Cytochrome C Oxidase Subunit 4I1 (Cox4i1) and elongation factor 2 (Eef2) (Fig. 4A). The heatmaps of these 19 common upregulated and downregulated genes were generated, with
Table I. Top 10 differentially expressed genes of the MAT1 group compared with the CONT group.

A, Upregulated genes

\begin{tabular}{lrr}
\hline Gene name & \multicolumn{1}{c}{ FC } & P-value \\
\hline Saa3 & 2847.6775 & 0.0012 \\
Lcn2 & 304.3738 & 0.0109 \\
RP23-459L15.8 & 47.7720 & 0.0012 \\
Egfros & 46.0328 & 0.0021 \\
RP23-380F8.2 & 44.2728 & 0.0194 \\
Nos2 & 43.0737 & 0.0023 \\
Fam71f2 & 40.7737 & 0.0026 \\
Gtf2a11 & 39.3699 & 0.0043 \\
RP24-111C16.1 & 37.2839 & 0.0125 \\
\hline
\end{tabular}

B, Downregulated genes

\begin{tabular}{lcc}
\hline Gene name & FC & P-value \\
\hline Yif1b & 0.0348 & 0.0173 \\
Rp123a-ps1 & 0.0295 & 0.0053 \\
Rps2-ps13 & 0.0284 & 0.0299 \\
RP24-574O8.7 & 0.0264 & 0.0023 \\
Hes1 & 0.0247 & 0.0043 \\
Ft12 & 0.0199 & 0.0376 \\
Ppp1ca & 0.0193 & 0.0307 \\
Nme2 & 0.0165 & 0.0051 \\
Nr4a1 & 0.0246 & 0.0215
\end{tabular}

FC, fold-change; CONT, control group; MAT1, ceramic disc in vitro group.

blue indicating lower expression (TPM $\leq 5$ ) and red indicating higher expression (TPM $>5$ ) (Fig. 4B). The top 10 continuously elevated genes were included in a trend graph (Fig. 4C).

Further GO analysis of trend genes revealed that the main enriched pathways were 'cell chemotaxis', 'negative regulation of ossification' and 'bone development pathway' (Fig. 5).

\section{Discussion}

Articular cartilage repair is a prominent focus of current research and represents a problem that needs to be resolved in clinical practice. The current experiments involve seed cells cultured in vitro and conventional soft scaffold structures that cannot be regulated and have biomechanical problems, which hinder the transformation of experimental results into clinical applications. It is crucial to prepare biodegradable scaffolds with osteoinductive properties; their application can provide a 3D environment for BMSCs at the site of the defect, where they can promote angiogenesis and thus contribute to the healing process (22). The biological materials currently used for bone repair include medical bioceramics, medical polymer materials, medical composite materials and artificial nanobone (23). 
Table II. Top 10 differentially expressed genes of the MAT2 group compared with the CONT group.

A, Upregulated genes

\begin{tabular}{lrr}
\hline Gene name & \multicolumn{1}{c}{ FC } & P-value \\
\hline Saa3 & 2584.2307 & 0.0037 \\
Lcn2 & 158.9995 & 0.0130 \\
Saa1 & 66.5714 & 0.0167 \\
Saa2 & 62.8477 & 0.0043 \\
Cp & 48.7990 & 0.0084 \\
Nos2 & 44.6269 & 0.0083 \\
Cxc15 & 41.3231 & 0.0220 \\
Dcn & 38.1207 & 0.0004 \\
Mmp3 & 37.9426 & 0.0004 \\
\hline
\end{tabular}

B, Downregulated genes

\begin{tabular}{lcc}
\hline Gene name & FC & P-value \\
\hline Slc34a2 & 0.0675 & 0.0263 \\
Fos & 0.0660 & 0.0003 \\
Rps2 & 0.0643 & 0.0067 \\
Hist1h2af & 0.0479 & 0.0461 \\
Cdsn & 0.0439 & 0.0147 \\
Spon2 & 0.0368 & 0.0040 \\
Wnt4 & 0.0287 & 0.0097 \\
Dlk2 & 0.0234 & 0.0099 \\
Nr4a1 & 0.0221 & 0.0243 \\
\hline FC, fold-change; CONT, control group; MAT2, ceramic rod in vitro \\
group.
\end{tabular}

In the present study, based on the microstructure characteristics of the hard bioceramic scaffold, the bioceramic scaffold was used to implant into the articular cartilage defect in mice. The scaffold suitable for microstructure absorbs autologous bone marrow cells and proliferates in the scaffold to repair articular cartilage. Seed cells do not need to be cultured in vitro, and the experimental results may provide a basis for clinical application. Since BMSCs and their differentiation during remodeling processes have essential roles in bone regeneration, it is thought that understanding the molecular signaling pathways involved is crucial to the development of bone implants, bone substitute materials and cell-based scaffolds for bone regeneration (24). The present study cultured and implanted BMSC scaffolds into mice and used RNA sequencing to explore the molecular mechanism of the bioceramic scaffolds according to the scaffold shape and duration of implantation.

PC1 level analysis showed that the measurements were reproducible, and the ceramic scaffold culture was better compared with the CONT group. The outcome for the MAT2 group was better compared with the MAT1 group; HCA results revealed the DEGs of each group. The MAT2 rod material is easier to implant compared with the MAT1 disc and has a
Table III. Top 10 differentially expressed genes in the MAT1 and MAT2 groups.

A, Upregulated genes

\begin{tabular}{lcc}
\hline Gene name & FC & P-value \\
\hline Ndufb6 & 36.7160 & 0.0245 \\
RP24-574O8.7 & 22.5928 & 0.0039 \\
Rpl17-ps9 & 20.2378 & 0.0356 \\
Rpl23a-ps1 & 18.4759 & 0.0090 \\
Mrp124 & 14.4720 & 0.0260 \\
Hspe1-ps2 & 14.2868 & 0.0333 \\
Ccl7 & 13.0649 & 0.0221 \\
Mrps36-ps2 & 12.4257 & 0.0297 \\
RP24-272N10.4 & 11.2402 & 0.0132 \\
Fth-ps2 & 10.6130 & 0.0475 \\
\hline
\end{tabular}

B, Downregulated genes

\begin{tabular}{lcc}
\hline Gene name & FC & P-value \\
\hline Tmem74b & 0.0797 & 0.0383 \\
Cacna1g & 0.0792 & 0.0152 \\
Nr4a3 & 0.0691 & 0.0005 \\
Crlf2 & 0.0649 & 0.0065 \\
Nr4a1 & 0.0643 & 0.0427 \\
Rnu3b4 & 0.0625 & 0.0050 \\
Il3ra & 0.0545 & 0.0071 \\
Fosb & 0.0454 & 0.0198 \\
Arrb2 & 0.0427 & 0.0045 \\
Sbf1 & 0.0376 & 0.0353 \\
\hline
\end{tabular}

FC, fold-change; MAT1, ceramic disc in vitro group; MAT2, ceramic rod in vitro group.

high degree of surface smoothness; this result can be used to optimize the clinical choices of bioceramics. By comparing the top 10 upregulated genes in the MAT1 group compared with the CONT group and in the MAT2 group compared with the CONT group, the intersection genes were found to be Saa3, Lcn2 and Nos2. Saa3 is a secreted protein that is prominently expressed in bone cells and affects bone metabolism by regulating genes expressed during inflammation, apoptosis and bone matrix remodeling (25). Saa3 may have a number of different biological functions related to extracellular matrix repair, bone remodeling, bone resorption and bone development (25). LCN2 is secreted by osteoblasts and is involved in bone metabolism, which is essential for bone health (26). Nos2 is elevated in osteoblasts and chondroblasts in bones during the early stages of fracture healing (27). Therefore, the bioinformatics analysis performed in the current study suggests that ceramic materials may be beneficial for bone formation.

Further comparison of the DEGs in the MAT2 and MAT1 groups revealed that the expression levels of osteogenesis-related genes, such as Ndufb6, Rpl17-ps9 and Ccl7, were upregulated. Ndufb6 is a component of the human skeletal 
A

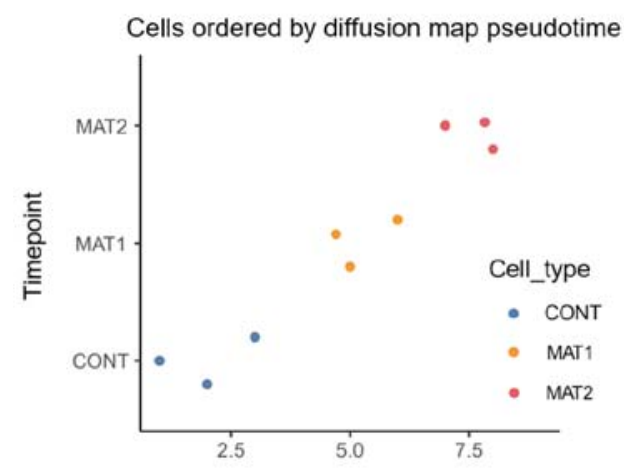

Diffusion map pseudotime

(first diffusion map component)
B

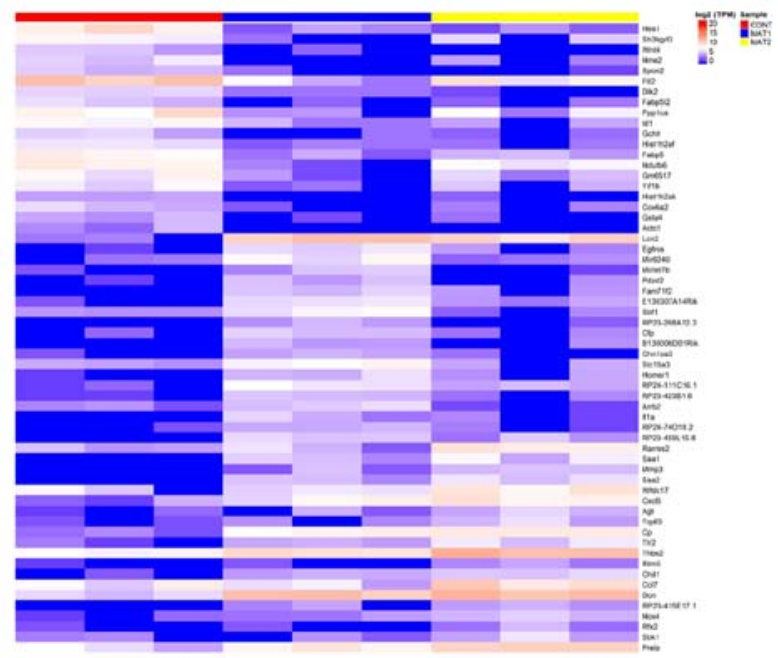

Figure 2. Principal components analysis and characteristic gene analysis. (A) Principal component 1 level analysis showed that the samples in each experimental group were reproducible, and the ceramic scaffold culture was superior to that of the control group. The MAT2 group had better reproducibility compared with the MAT1 group. (B) Hierarchal clustering analysis map showing the characteristic genes in each group. CONT, control group; MAT1, ceramic disc in vitro group; MAT2, ceramic rod in vitro group.

A

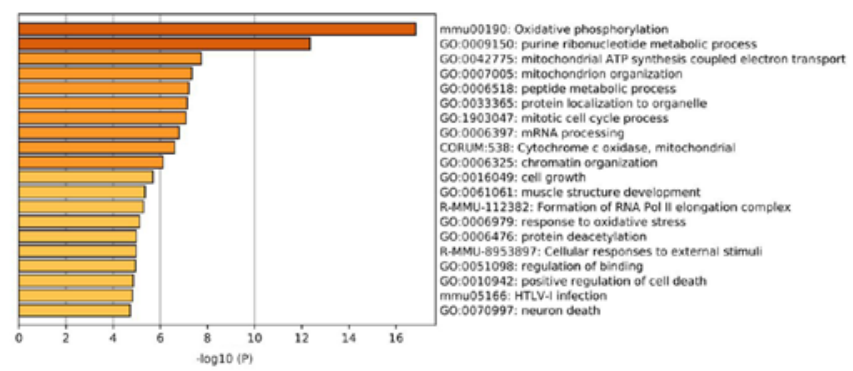

B

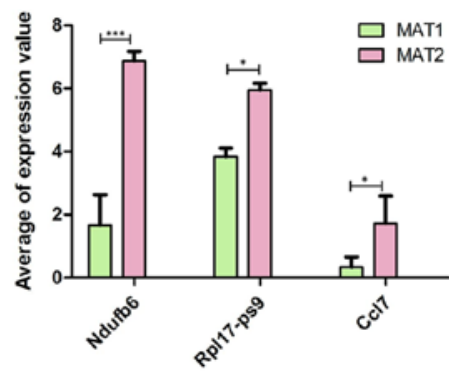

Figure 3. MAT2-MAT1 differentially expressed gene analysis. (A) GO analysis of differentially expressed genes between the MAT1 and MAT2 groups. (B) Comparison of Ndufb6, Rp117-ps9 and Cc17 gene expression in the MAT1 and MAT2 groups. ${ }^{*} \mathrm{P}<0.05$ and ${ }^{* * *} \mathrm{P}<0.001$. GO, Gene Ontology; MAT1, ceramic disc in vitro group; MAT2, ceramic rod in vitro group; Ndufb6, NADH: Ubiquinone oxidoreductase subunit B6; Rp117-ps9, ribosomal protein L17, psudogene 9; Ccl7, C-C motif chemokine ligand 7.

muscle respiratory chain (28). Rpl17 can be used in the early stages of osteogenic differentiation in mouse bone marrow mesenchymal stem cells (29). CCL7 increases the mRNA levels of a number of genes involved in metastasis and osteolysis (30). Therefore, it was hypothesized that the material used in the MAT2 group may be superior to that used in the MAT1 group. Therefore, rod-shaped ceramic implants used in the MAT2 group were selected for subsequent in vivo experiments.

The ceramic rods were implanted into mice for 3 or 6 days. By comparing the DEGs of each group, it was found that there were 10 genes with increased expression levels, such as Ammecrll, Tnfaip2, Ikbke and Prkcd. Nine genes, including Fdx1, Igfbp6, Banf1 and Eef2 showed decreased expression levels. Further GO analysis of the 19 DEG expression trends revealed that the main pathways involved were 'cell chemotaxis', 'negative regulation of ossification' pathway and 'bone development'. The involvement of the important signaling pathways associated with the bone development during embryogenesis, as well as during fracture healing and repair has been demonstrated by various studies. These include the Wnt//-catenin, Notch, bone morphogenic protein/transforming growth factor- $\beta$, PI3K/Akt/mTOR, mitogen-activated protein kinase, platelet-derived growth factor, insulin-like growth factor, fibroblast growth factor and $\mathrm{Ca}^{2+}$ pathways (24). It has been suggested that BMSCs and their differentiation during the remodeling processes, as well as specific signaling pathways and their activated downstream networks, are implicated in bone regeneration (31). The present study indicated that the BMSC ceramic rod was successfully implanted into the mouse and played a role in repairing bone damage. The bone repair effect was more obvious as the implantation time was increased. Numerous treatment strategies can improve bone repair, which opens up new avenues for bone regeneration therapies. One of the strategies is based on the study of molecular signaling pathways. Changing gene expression levels to increase the number of osteoblasts or promote their maturity is expected to stimulate bone regeneration. The experimental limitation of the present study is the lack of quantitative PCR and western blot validation data, which will be performed in subsequent studies.

The present study successfully performed three-dimensional culture of mesenchymal stem cell composite scaffolds in mice and used RNA sequencing to explore its molecular mechanism based on the shape of bioceramic scaffold and the duration of implantation in vivo. Molecular studies found that rod-shaped ceramic materials were superior to the disc shape. 


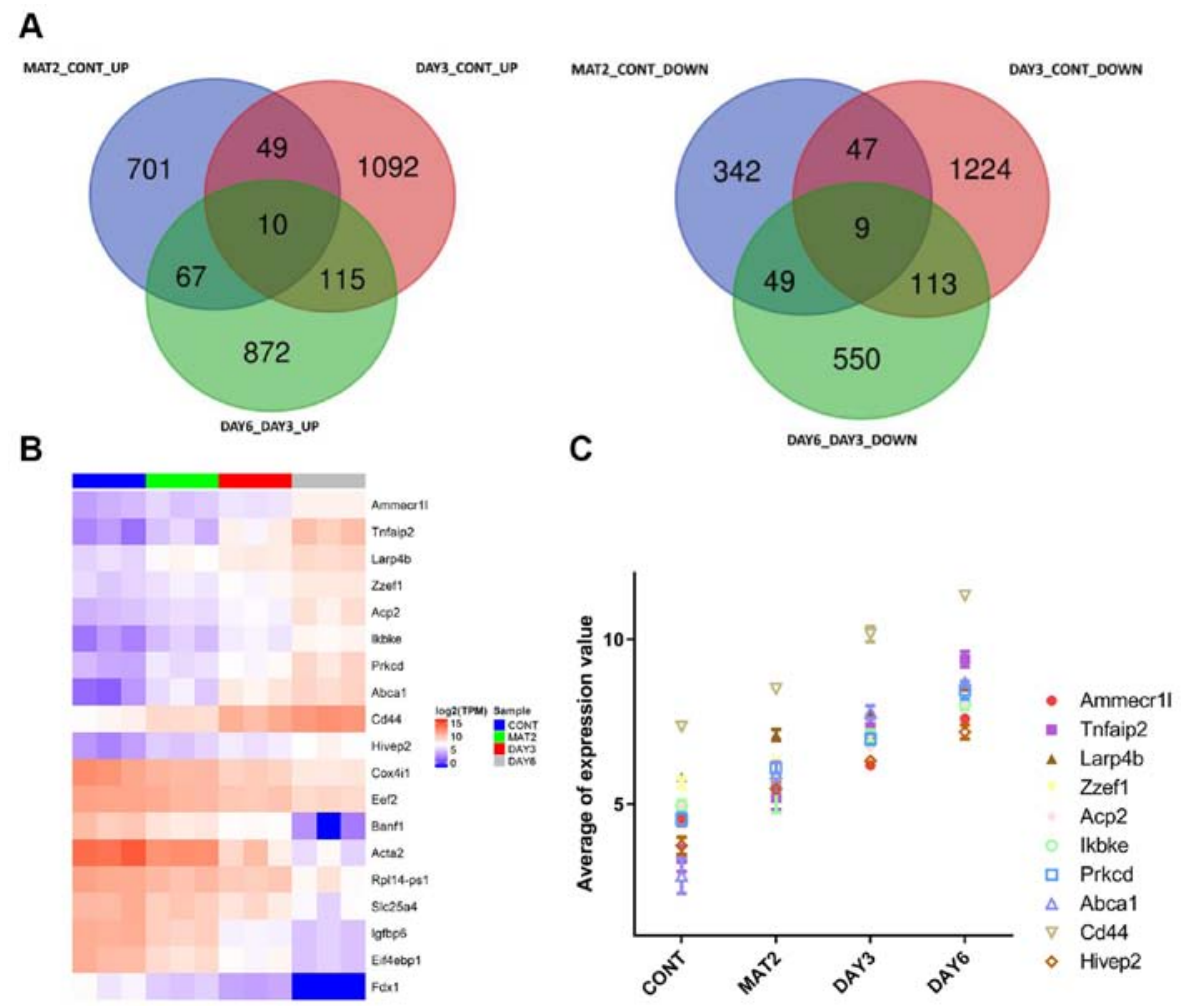

Figure 4. Gene expression trend analysis. (A) Venn diagram showing upregulated and downregulated differentially expressed genes of MAT2 vs. CONT, DAY3 vs. CONT and DAY6 vs. DAY3. (B) Heat map showing trend changes in CONT-MAT2-DAY3-DAY6 genes. (C) Trend chart of top 10 genes with continually increased expression.

A

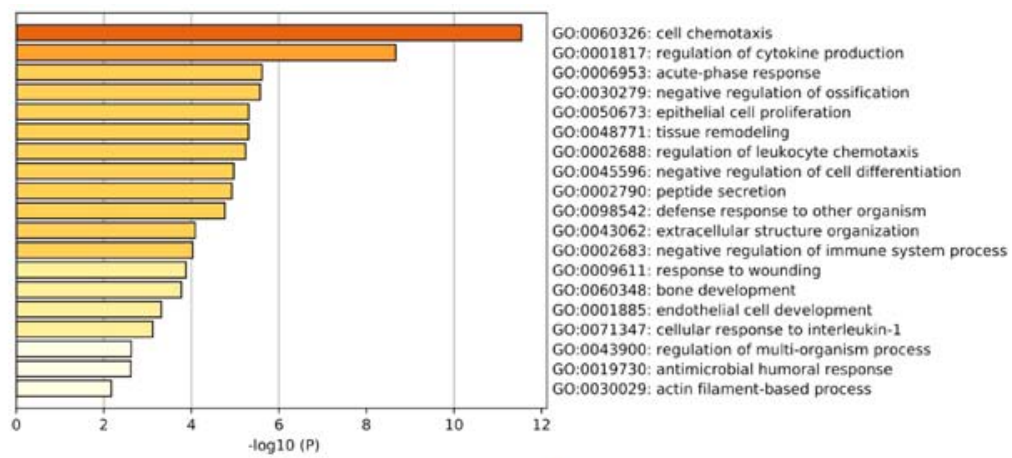

8

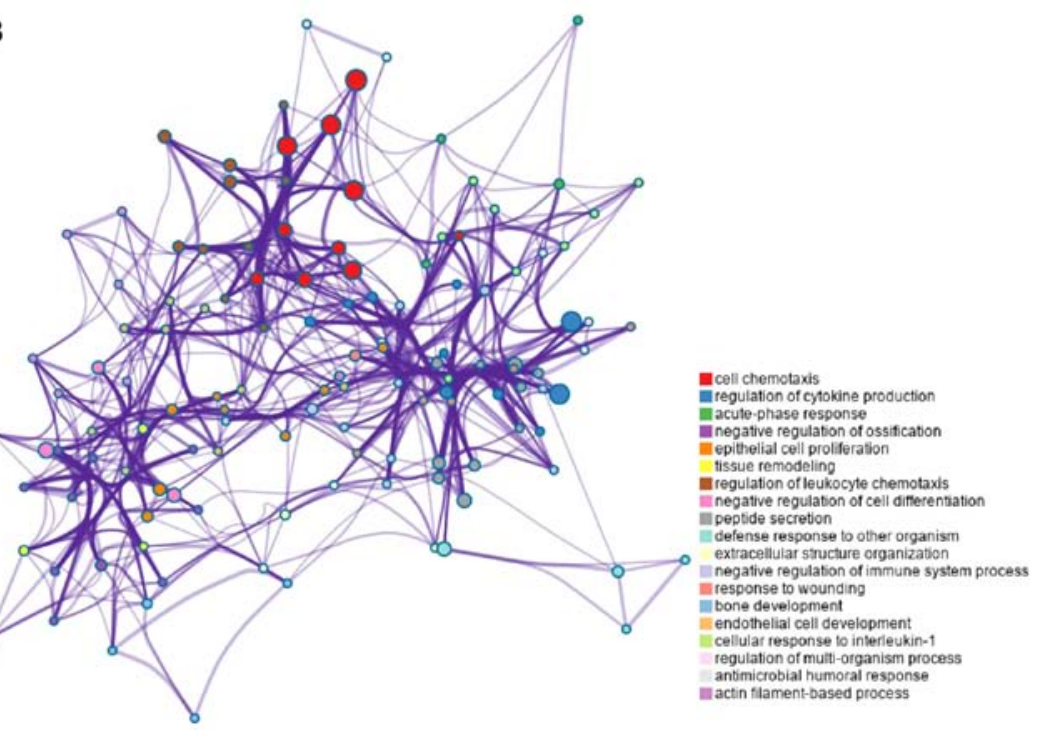

Figure 5. GO analysis and interaction network of the gene trends (upregulated and downregulated differentially expressed genes of CONT, DAY3 vs. CONT and DAY6 vs. DAY3.). GO, Gene Ontology. (A) GO analysis of trend genes revealed the main enriched pathways. (B) Trend gene interaction network. 
This conclusion may be used to optimize clinical choices for bioceramics. By comparing the DEGs of each group, it was further confirmed that BMSCs were suitable as seed cells for cartilage tissue engineering, and the $\beta$-TCP scaffold may be ideal cartilage tissue engineering scaffold material. The present research provided new insights into the molecular mechanism of cartilage repair by BMSCs and bioceramic scaffolds.

\section{Acknowledgements}

The authors would like to acknowledge Dr Xunxia Bao and Dr Sibo Zhu of (CinoAsia Co., Ltd.) for their technical assistance and editing of the text.

\section{Funding}

The current study was supported by The National Natural Science Foundation of China (grant no. 81572128). Shanghai Municipal Commission of Health and Family Planning (grant no. 201940170).

\section{Availability of data and materials}

The datasets used and/or analyzed during the present study are available from the corresponding author on reasonable request.

\section{Authors' contributions}

$\mathrm{XH}$ and ZC conceptualized the study, designed the research and performed the experiments. GZ and JS performed the experiments. GH, FC, YW and JX analyzed and interpreted the data. JC and SW designed the experiments, analyzed and interpreted the data, wrote and edited the manuscript, and supervised the project. All authors read and approved the final manuscript.

\section{Ethics approval and consent to participate}

All experiments were performed according to the principles outlined in the Guide for the Care and Use of Laboratory Animals and the current study was approved by the Ethics Committee of Huashan Hospital Affiliated to Fudan University (approval no. 2018 Huashan Hospital JS-098).

\section{Patient consent for publication}

Not applicable.

\section{Competing interests}

The authors declare that they have no competing interests.

\section{References}

1. Huang Z, Ding C, Li T and Yu SPC: Current status and future prospects for disease modification in osteoarthritis. Rheumatology (Oxford) 57 (Suppl 4): iv108-iv123, 2018.

2. Abramson SB, Attur M and Yazici Y: Prospects for disease modification in osteoarthritis. Nat Clin Pract Rheumatol 2: 304-312, 2006

3. Karsdal MA, Leeming DJ, Dam EB, Henriksen K, Alexandersen P, Pastoureau P, Altman RD and Christiansen C: Should subchondral bone turnover be targeted when treating osteoarthritis? Osteoarthritis Cartilage 16: 638-646, 2008.
4. Ito Y, Ochi M, Adachi N, Sugawara K, Yanada S, Ikada Y and Ronakorn P: Repair of osteochondral defect with tissue-engineered chondral plug in a rabbit model. Arthroscopy 21: 1155-1163, 2005.

5. Ebihara G, Sato M, Yamato M, Mitani G, Kutsuna T, Nagai T, Ito S, Ukai T, Kobayashi M, Kokubo M, et al: Cartilage repair in transplanted scaffold-free chondrocyte sheets using a minipig model. Biomaterials 33: 3846-3851, 2012.

6. Sivasubramaniyan K, Ilas DC, Harichandan A, Bos PK, Santos DL, de Zwart P, Koevoet WJLM, Owston H, Bühring HJ, Jones E and van Osch GJVM: Bone marrow-harvesting technique influences functional heterogeneity of mesenchymal stem/stromal cells and cartilage regeneration. Am J Sports Med 46: 3521-3531, 2018.

7. Otsuru S, Tamai K, Yamazaki T, Yoshikawa H and Kaneda Y: Bone marrow-derived osteoblast progenitor cells in circulating blood contribute to ectopic bone formation in mice. Biochem Biophys Res Commun 354: 453-458, 2007.

8. Li H, Dai K, Tang T, Zhang X, Yan M and Lou J: Bone regeneration by implantation of adipose-derived stromal cells expressing BMP-2. Biochem Biophys Res Commun 356: 836-842, 2007.

9. Hayashi $\mathrm{O}$, Katsube $\mathrm{Y}$, Hirose $\mathrm{M}$, Ohgushi $\mathrm{H}$ and Ito $\mathrm{H}$ : Comparison of osteogenic ability of rat mesenchymal stem cells from bone marrow, periosteum, and adipose tissue. Calcif Tissue Int 82: 238-247, 2008.

10. Pitchford SC, Furze RC, Jones CP, Wengner AM and Rankin SM: Differential mobilization of subsets of progenitor cells from the bone marrow. Cell Stem Cell 4: 62-72, 2009.

11. de Girolamo L, Lucarelli E, Alessandri G, Avanzini MA, Bernardo ME, Biagi E, Brini AT, D'Amico G, Fagioli F, Ferrero I, et al: Mesenchymal stem/stromal cells: A new 'cells as drugs' paradigm. Efficacy and critical aspects in cell therapy. Curr Pharm Des 19: 2459-2473, 2013.

12. Oryan A, Alidadi S, Moshiri A and Maffulli N: Bone regenerative medicine: Classic options, novel strategies, and future directions. J Orthop Surg Res 9: 18, 2014.

13. Tuli R, Tuli S, Nandi S, Wang ML, Alexander PG, Haleem-Smith H, Hozack WJ, Manner PA, Danielson KG and Tuan RS: Characterization of multipotential mesenchymal progenitor cells derived from human trabecular bone. Stem Cells 21: 681-693, 2003.

14. Hernigou P and Beaujean F: Treatment of osteonecrosis with autologous bone marrow grafting. Clin Orthop Relat Res 14-23, 2002.

15. Qin X, Raj RM, Liao XF, Shi W, Ma B, Gong SQ, Chen WM and Zhou B: Using rigidly fixed autogenous tooth graft to repair bone defect: An animal model. Dent Traumatol 30: 380-384, 2014.

16. Korhonen RK and Jurvelin JS: Compressive and tensile properties of articular cartilage in axial loading are modulated differently by osmotic environment. Med Eng Phys 32: 155-160, 2010.

17. Siqueira L, Passador FR, Costa MM, Lobo AO and Sousa E: Influence of the addition of $\beta$-TCP on the morphology, thermal properties and cell viability of poly (lactic acid) fibers obtained by electrospinning. Mater Sci Eng C Mater Biol Appl 52: 135-143, 2015.

18. Finkemeier CG: Bone-grafting and bone-graft substitutes. J Bone Joint Surg Am 84: 454-464, 2002.

19. Giannoudis PV, Dinopoulos H and Tsiridis E: Bone substitutes: An update. Injury 36 (Suppl 3): S20-S27, 2005.

20. Lu JX, Flautre B, Anselme K, Hardouin P, Gallur A, Descamps M and Thierry B: Role of interconnections in porous bioceramics on bone recolonization in vitro and in vivo. J Mater Sci Mater Med 10: 111-120, 1999.

21. Ikeda R, Fujioka H, Nagura I, Kokubu T, Toyokawa N, Inui A, Makino T, Kaneko H, Doita M and Kurosaka M: The effect of porosity and mechanical property of a synthetic polymer scaffold on repair of osteochondral defects. Int Orthop 33: 821-828, 2009.

22. Oryan A, Kamali A, Moshiri A and Baghaban Eslaminejad M: Role of mesenchymal stem cells in bone regenerative medicine: What is the evidence? Cells Tissues Organs 204: 59-83, 2017.

23. Kashte S, Jaiswal AK and Kadam S: Artificial bone via bone tissue engineering: Current scenario and challenges. Tissue Eng Regen Med 14: 1-14, 2017.

24. Majidinia M, Sadeghpour A and Yousefi B: The roles of signaling pathways in bone repair and regeneration. J Cell Physiol 233: 2937-2948, 2018 
25. Thaler R, Sturmlechner I, Spitzer S, Riester SM, Rumpler M, Zwerina J, Klaushofer K, van Wijnen AJ and Varga F: Acute-phase protein serum amyloid A3 is a novel paracrine coupling factor that controls bone homeostasis. FASEB J 29: 1344-1359, 2015.

26. Capulli M, Ponzetti M, Maurizi A, Gemini-Piperni S, Berger T, Mak TW, Teti A and Rucci N: A complex role for lipocalin 2 in bone metabolism: Global ablation in mice induces osteopenia caused by an altered energy metabolism. J Bone Miner Res 33: 1141-1153, 2018.

27. Huang W, Zhang K, Zhu Y, Wang Z, Li Z and Zhang J: Genetic polymorphisms of NOS2 and predisposition to fracture non-union: A case control study based on han Chinese population. PLoS One 13: e0193673, 2018.

28. Kacerovsky-Bielesz G, Chmelik M, Ling C, Pokan R, Szendroedi J, Farukuoye M, Kacerovsky M, Schmid AI, Gruber S, Wolzt M, et al: Short-term exercise training does not stimulate skeletal muscle ATP synthesis in relatives of humans with type 2 diabetes. Diabetes 58: 1333-1341, 2009.
29. Hermann-Kleiter N, Ghaffari-Tabrizi N, Blumer MJ, Schwarzer C, Mazur MA and Artner I: Laspl misexpression influences chondrocyte differentiation in the vertebral column. Int J Dev Biol 53: 983-991, 2009.

30. Han S, Wang T, Chen Y, Han Z, Guo L, Wu Z, Yan W, Wei H, Liu T, Zhao J, et al: High CCL7 expression is associated with migration, invasion and bone metastasis of non-small cell lung cancer cells. Am J Transl Res 11: 442-452, 2019.

31. Knight MN and Hankenson KD: Mesenchymal stem cells in bone regeneration. Adv Wound Care (New Rochelle) 2: 306-316, 2013.

(7) (9) This work is licensed under a Creative Commons Attribution-NonCommercial-NoDerivatives 4.0 International (CC BY-NC-ND 4.0) License. 